\title{
Developing Competence in Tooth Extractions during Undergraduate Dental Education - A Qualitative Study
}

\author{
Ali, K. ${ }^{1}$, Siddiqi, K.M. ${ }^{2}$, Qazi, H.S. ${ }^{2}$
}

\begin{abstract}
Purpose: To gain a deeper understanding of factors which contribute to development of competence in tooth extractions during undergraduate dental education.

Methods: A qualitative approach was used to explore the perceptions and experiences of a range of stakeholders. Purposive sampling was used to recruit the participants which included undergraduate dental students, new graduates, dental academic staff, and clinical supervisors. The focus groups were transcribed verbatim, and data were imported into NVivo 12. Data were analyzed thematically using an inductive approach.
\end{abstract}

Results: Two focus groups were conducted. Ten undergraduate students participated in the Student Focus Group while eight dental academics and clinical supervisors participated in the Staff Focus Group. Participants expressed their views on a range of issues related to teaching and training of tooth extractions. The key themes cantered on structured teaching underpinned by patient safety; focus on breadth of clinical experience; and constructive feedback to enhance reflective practice amongst students to enhance the learning experiences of students.

Conclusions: This study provides useful insights into how undergraduate dental students develop competence in tooth extractions as perceived by the participants and may help dental educators to reflect of their teaching model.

Keywords: Clinical Skills, Oral and Maxillofacial Surgery, Undergraduate Dental, Competence, Extractions

\section{Introduction}

Competence in tooth extractions is a core skill in undergraduate dental programs. Students are expected to be proficient in performing uncomplicated extractions prior to graduation (GDC, 2015; Cowpe et al, 2010). Competence of new dental graduates is defined by abilities essential to begin independent, unsupervised dental practice (Chambers \& Gerrow, 1994).

${ }^{1}$ Qatar University College of Dental Medicine, Qatar

${ }^{2}$ Islamabad Medical and Dental College, Pakistan

Corresponding author: Professor Kamran Ali

ali.kamran@qu.edu.qa
However, competency of a new dental graduate like their medical peers only represents a point on a continuum (Harden, 2007; Frank et al., 2010).

A newly qualified dentist usually develops through a predictable pattern after experiencing a variety of challenges in the clinical environment and it may take several years to consolidate their knowledge, skills, and attitudes (Chambers, 1996).

Several studies investigating dental students' competence in tooth extractions have been published in recent years (Al-Dajani, 2015; Macluskey \& Durham, 2009; Durham et al., 2007).

DOI: http://doi.org/10.4038/seajme.v15i2.299 
Evidence from the literature underscores the need to improve students' training in performing tooth extractions for a smooth transition into general dental practice settings (Wilson, 2011). Dental institutions, especially those in the developed countries, have adopted a competency-based framework for imparting dental education (Prescott et al., 2001). However, marked differences can be identified in the structure of teaching, clinical training, and assessments of tooth extractions in dental schools globally (Brand et al., 2015). Similarly, clinical targets in tooth extractions during undergraduate education vary considerably ranging from as low as 20 in the United Kingdom to 200 in some developing countries (Durham et al., 2007; HEC, 2011). Controversies also exist regarding the relationship between the total number of teeth extracted and the successful completion of the final assessment (Ali et al., 2020; Al-Dajani, 2015). Therefore, further investigation of factors, which contribute to development of students' competency in tooth extractions, is warranted.

The aim of the study was to explore the development of competence in tooth extractions during undergraduate dental education and gain a deeper understanding of factors which facilitate acquisition of knowledge, as well as psychomotor and affective skills to help dental students undertake tooth extractions on patients in a safe and predictable manner.

The conceptual framework of the study was underpinned by situated learning theory (Lave \& Wenger, 1991). Situated learning asserts that learning is a transformative process tied to its context and to the social relations and practices. Exposure of learners to authentic situations and activities facilitates learning (Herrignton \& Oliver, 2000). In the context of clinical dental education, students and academics come together in pursuit of a shared enterprise. Through "legitimate peripheral participation", active engagement and assuming increasing responsibility, the student in this session acquired the role, skills, and values of the culture and community (Lave \& Wenger, 1999).

\section{Methods}

Ethical approval was obtained for the study from the Institutional Review Board (IMDC/DS/IRB/164). This study was undertaken at the Islamabad Medical and Dental College, Islamabad Pakistan. Participants were recruited using purposive sampling to target undergraduate students, dental academic staff and clinical supervisors. Qualitative methods were used for data collection and two focus groups were organized: one for students, and the other one for staff. The participants were invited by email through the central Faculty office with the Administration officer as the gatekeeper. The email invites were accompanied by a participant information sheet explaining the purpose and scope of the study and other relevant details regarding data collection, storage, and dissemination along with contact details of the research team. Informed consent was obtained from all participants to confirm their agreement to participate in the study. The participants were offered a choice of dates and times separately and asked to indicate their preferences through a doodle poll. Based on the responses, a date, time, and location were worked out for each focus group and communicated to the participants by email.

Participants in the Staff focus group were dental academics, and clinical supervisors. The second focus group consisted of final-year dental students and recent graduates. Both focus groups were facilitated by the principal researcher who had no previous direct contact with the participants. A separate focus group for students was aimed at allowing them to share their perceptions and experiences as presence of staff could be potentially intimidating.

Both focus groups were recorded on a digital audio device to ensure accuracy of data collection and allow the research team to revisit the original data during analyses. The focus group recordings were transcribed verbatim by the research team within two weeks of data collection. The transcripts were anonymized using pseudonyms to protect the identity of the participants. The typed transcripts of focus groups were shared with participants of each 
group to confirm their accuracy. The data were imported into NVivo 12 (QSR International Pty. Ltd., Doncaster, Victoria, Australia).

The process of data analysis was started by listening to the audio recordings repeatedly along with reading the transcripts. Deep engagement with the data was essential for situating competence in tooth extractions within the narratives of stakeholders emanating from their understanding, experiences, and expectations. Data analysis focused on capturing the context and meanings of the rich imagery shared by the participants. Systematic reading through the entire data set was used for an initial coding. Repetitive revisiting of the data helped to collapse the nodes into broader codes. Further analysis and reflection helped to establish linkages between nodes, which facilitated development of tree nodes from free nodes and ultimately helped shape the themes.

An inductive approach was used for thematic analysis i.e., the themes were data driven Segments of verbatim quotes from different participants were incorporated as coded text to provide the contextual material supporting different themes. Member checking was used to validate the data analysis. Two colleagues who were experienced qualitative researchers compared analysis to verify the accuracy of the coding and categorization of themes. Member checking resulted in minor modifications to categorization of data under different themes. The results were shared with two participants from each of the focus groups to determine the interpretation of the data was consistent with their views.

\section{Results}

Eighteen participants were involved in this study. The student focus groups had ten participants from the final year (six females and four females). The staff focus group had eight participants (six males and two females). Participants shared their perceptions and experiences regarding a range of issues related to teaching, clinical training, assessments, and supervisor feedback on tooth extractions. The participants also provided recommendations to enhance the learning experiences of students.
The key themes emanating from data analysis where there was a broad agreement amongst the participants are summarized below.

\section{Patient Safety}

Participants in both groups unanimously expressed the need to place patient safety at the heart of teaching and training in tooth extractions. They emphasized the need for comprehensive and meticulous preoperative evaluation of patients' medical history, clinical and radiographic assessment to allow recognition of potential difficulties and complications.

"Students often find it difficult to recognize how patient's medical conditions and drug prescriptions can complicate tooth extractions and we need to focus more on these aspects in our teaching". Clinical supervisor 2

Routine use of surgical safety checklists was recognized as a mandatory step to prevent extraction of a wrong tooth; this was particularly highlighted by the participants in the staff focus group. Most student participants also reported their concerns regarding patient safety and felt more comfortable when they were observed directly by their clinical supervisors.

"I was very nervous and somewhat scared that I might harm my patient or take out the wrong tooth and having my clinical supervisor observe me while performing the extraction was reassuring". Dental Student 3.

Regarding operative skills in tooth extractions, structured training supported by bespoke video resources followed by practical training and summative assessments in simulated environment were also identified as an essential requirement prior to students being allowed to undertake tooth extractions on patients.

"Learning tooth extractions on mannequin can be very helpful to understand patient loperator position, tooth movements with forceps and the use of non-dominant hand, you can focus on each step, it is more challenging to do so when working on real patients". Dental Student 5 
Student participants unanimously expressed their desire to start learning tooth extractions in earlier stages of the program rather than in the pre-final year as it would allow them more time to consolidate their clinical skills; also shadowing specialists undertaking difficult extractions was considered to be beneficial to boost their confidence.

\section{Breadth of Clinical Experience}

Participants in the staff and students' groups felt that the breadth of clinical experience was more relevant than the numerical clinical targets. Students felt that the signing off students purely on the basis of number of extractions could sometimes be misleading especially when based on extraction of periodontally involved teeth and mobile, retained roots localized to the soft tissues. Students also mentioned lack of confidence in undertaking tooth extractions involving medically compromised patients due to limited experience.

Variations in difficulty level of individual teeth could also impact on the competence and confidence of students and dental trainers should focus on providing a broad-based clinical experience in extractions to students. Four students always extractions of upper first molars to be challenging due to the complex root morphology and reiterated the need for additional opportunities to extract these teeth.

"I find extraction of upper first molars most difficult and need more experience to feel confident". Dental Student 7

Participants in the staff focus group also acknowledged the importance of breadth of clinical experience. However, resource implications for a comprehensive triage of suitable patients on student clinics were considered a barrier by staff as it entailed additional administrative and clinical input.

Individualized and immediate feedback on student performance in simulated and clinical settings was identified as a key factor in enhancing student competence in tooth extractions and this was endorsed by all participants uniformly. Student participants valued timely and constructive feedback and acknowledged self-reflection was crucial for developing their skills in tooth extractions during their university education and beyond.

"I was not confident with my extraction but feedback and support from my supervisors helped me identify and reflect at each patient encounter, now I am more comfortable taking teeth out" Dental Student 1

One student mentioned that they had received inconsistent feedback and strong comments from their supervisor which had affected their confidence adversely.

Participants in the staff group mirrored students' comments regarding the importance of feedback and reflective practice. However, three supervisors highlighted that feedback was a two-way process and students who actively seek feedback are more likely to benefit from it.

"Students need to engage more actively with feedback from their tutors, they need to be receptive to criticism and ask for areas of improvement, there is always room to do things better". Clinical Supervisor 5

It was also reiterated that the feedback should not be restricted to assessments only and should be provided for every patient encounter involving tooth extraction. Recording students' performance for all patient encounters would also generate sufficient data point to monitor and evaluate student skill development longitudinally facilitating timely remediation.

\section{Discussion}

This is perhaps the first study qualitative study exploring development of tooth extraction competency in undergraduate dental education and provides helpful insights into the processes involved in student training. Patient safety is an overarching phenomenon, which impacts on all aspects of clinical education and must be prioritized throughout. The National Academy of Medicine in the United States in its report entitled 'To err is human' highlighted the 
importance of human factors, which may contribute to errors in clinical services (Kohn et al., 2000). Human errors during tooth extractions are multifactorial including time constraints, pre-operative assessment, managing patient anxiety, identification of correct teeth, difficulties in accessing the operative site. Given that tooth extractions are performed routinely, it is one of the most common procedure associated with wrong site surgery (WSS). Like other healthcare providers, dental schools in Western countries are increasingly implementing surgical safety checklists on student clinics to mitigate against WSS but it is not possible to ascertain if this is being implemented in underdeveloped countries consistently (Dargue et al., 2020).

Comprehensive assessment and preparation of medically compromised patients for invasive procedures is also essential for safe clinical practice. However, given the variations in teaching and training models in dental schools, dental students may not always be competent to manage patients with underlying health conditions (Chávez et al., 2020). Some schools may address this by filtering medically compromised patients from student clinics. However, there is a risk that lack of exposure to patients with additional healthcare needs may impact adversely on confidence of students, which may extend into their clinical careers after graduation (Holzinger et al., 2020).

Appropriate staff/student ratio for supervision of tooth extractions is also essential for a number of reasons including, patient safety, quality of learning experience, and supervisor feedback. An staff/student ratio of 1:5 has been reported in the literature from the United Kingdom, but recent recommendations suggest that ideally a ratio of $1: 3$ is required to ensure patient safety and student experience (Dargue et al., 2020). It is acknowledged that this target may be ambitious for some schools, especially in the face of faculty shortages in dental schools (Hamamoto et al., 2013).

Variations in curricular design and teaching models in dental schools affect the stage at which tooth extraction teaching is introduced in the dental programs. Schools following traditional curricula tend to compartmentalize basic sciences and clinical disciplines and students need to wait two to three years before seeing patients. Early clinical exposure is more likely to allow dental students more time to develop their clinical skills in addition to immense opportunities to their skills in team working, communication, and professionalism in clinical environments (Ali et al., 2018). Given that tooth extraction constitutes an irreversible procedure, dental schools must endeavor to provide structured training and standardized summative assessments in simulated environments prior to allowing them to undertake tooth extractions on patients (Brand et al., 2015). Moreover, teaching should be supported by high quality video resources tailored to the stage of student learning, as reiterated by participants in this study. This may be particularly relevant to underdeveloped countries where practical training in simulated learning may be deficient due to limited resources (Ali et al., 2020).

Participants in this study emphasized the importance of the breadth of clinical experience as numbers alone may not provide an accurate measure of their skills in performing tooth extractions. These findings corroborate with previous studies on factors influencing clinical competence of dental students (Dawson et al., 2017; Stelzle et al., 2011). Dental educators need to ensure that students gain experience in extracting all types of teeth in both arches to account for variations in access, root morphology, bone thickness, relationship to adjacent anatomical structures (Hupp et al., 2008).

Clinical assessments of competence in tooth extractions may be complicated by difficulties in standardizing patients and therefore, assessments should be carried out longitudinally over a sustained period of time, using multiple data points rather than on a single assessment occasion. This approach would also facilitate regular feedback to the students providing them with opportunities to reflect on their performance, and seek remediation, if required. It is recognized that recording of student performance for each patient encounter is time-consuming and would 
ideally require use of digital platforms, which may not be possible for all institutions due to resource constraints.

The main limitation of this study is that the participants were from a single institution. The perspectives and experiences of the participants in this study may be shaped by the educational environment in a developing country. Nevertheless, this research has identified several key areas which may impact on the development of students' competency in tooth extractions. Future collaborative studies involving multiple institutions from different geographic locations are recommended to inform the teaching and assessments of undergraduate students.

\section{Conclusion}

This study provides useful insights into how undergraduate dental students develop competence in tooth extractions as perceived by the stakeholders. Competence development may be facilitated by structured training in simulated and clinical settings incorporating patient safety, providing early clinical exposure with a focus on breath of clinical experience, developing a comprehensive patient triage system, close supervision and feedback by experienced supervisors, reflective practice, and periodic assessments to monitor students' performance longitudinally. The findings of this study may help dental educators to revisit their teaching on tooth extractions in undergraduate dental education programs.

\section{Acknowledgements}

The authors would like to thank all the participants for their contribution to this research.

\section{References}

Al-Dajani, M., 2015. Dental students' perceptions of undergraduate clinical training in oral and maxillofacial surgery in an integrated curriculum in Saudi Arabia. Journal of educational evaluation for health professions, 12.

Ali, K., Qazi, H.S., Siddiqi, K. and Glanville, R., 2021. Assessment of undergraduate students in tooth extraction competence-A cohort study. European Journal of Dental Education, 25(3), pp.607-613.
Ali, K., Zahra, D., McColl, E., Salih, V. and Tredwin, C., 2018. Impact of early clinical exposure on the learning experience of undergraduate dental students. European Journal of Dental Education, 22(1), pp.e75-e80.

Brand, H.S., Van Der Cammen, C.C., Roorda, S.M. and Baart, J.A., 2015. Tooth extraction education at dental schools across Europe. BDJ open, 1(1), pp.1-6.

Chambers, D.W. and Gerrow, J.D., 1994. Manual for developing and formatting competency statements. Journal of dental education, 58(5), pp.361-66.

Chambers, D.W., 1996. Dental curriculum and accreditation--means, ends, and the continuum. Journal of dental education, 60(10), pp.816-820.

Chávez, E.M., Subar, P.E., Miles, J., Wong, A., LaBarre, E.E. and Glassman, P., 2011. Perceptions of predoctoral dental education and practice patterns in special care dentistry. Journal of Dental Education, 75(6), pp.726-732.

Cowpe, J., Plasschaert, A., Harzer, W., VinkkaPuhakka, H., Walmsley, A.D. and Walmsley, A.D., 2009. Profile and competences for the European dentist-update 2009.

Dargue, A., Fyfe, E., French, K., Ali, K., Bailey, E., Bell, A., Bolt, R., Bulsara, Y., Carey, J., Emanuel, C. and Green, R., 2021. The impact of wrong-site surgery on dental undergraduate teaching: a survey of UK dental schools. European Journal of Dental Education, 25(4), pp.670-678.

Dawson, L.J., Mason, B.G., Bissell, V. and Youngson, C., 2017. Calling for a re-evaluation of the data required to credibly demonstrate a dental student is safe and ready to practice. European Journal of Dental Education, 21(2), p.130.

Durham, J.A., Moore, U.J., Corbett, I.P. and Thomson, P.J., 2007. Assessing competency in dentoalveolar surgery: a 3-year study of cumulative experience in the undergraduate curriculum. European Journal of Dental Education, 11(4), pp.200-207.

Frank, J.R., Snell, L.S., Cate, O.T., Holmboe, E.S., Carraccio, C., Swing, S.R., Harris, P., Glasgow, N.J., Campbell, C., Dath, D. and Harden, R.M., 2010. Competency-based medical education: theory to practice. Medical teacher, 32(8), pp.638-645.

GDC. 2015. Preparing for practice. Dental team learning outcomes for registration. London: General Dental Council. 
Hamamoto, D.T., Farrar, S.K., Caplan, D.J., Lanphier, T.F., Panza, J.C. and Ritter, A.V., 2013. Use of shared faculty in US and Canadian dental schools. Journal of dental education, 77(3), pp.264-275.

Harden, R. M. 2007. Outcome-based education: the future is today. Medical Teacher, 29 (7). pp 625629

Herrington, J. and Oliver, R., 2000. An instructional design framework for authentic learning environments. Educational technology research and development, 48(3), pp.23-48.

Higher Education Commission. Curriculum of Bachelor of Dental Surgery (BDS) Five-Year Programme, 2011. Available online at: https://hec.gov.pk/english/services/universities/ RevisedCurricula/Documents/2010-2011/DraftBDS-2011.pdf (Accessed June, 2021).

Holzinger, A., Lettner, S. and Franz, A., 2020. Attitudes of dental students towards patients with special healthcare needs: Can they be improved?. European Journal of Dental Education, 24(2), pp.243-251.

Hupp MR, Ellis JR, Tucker E.2008. Contemporary oral and maxillofacial surgery. Elsevier Missouri. 6th Edition: pp.92-112.
Kohn, L.T., Corrigan, J.M. and Donaldson, M.S. 2000. Errors in health care: a leading cause of death and injury. In To err is human: Building a safer health system. National Academies Press (US).

Lave J, Wenger E. 1991. Situated learning: legitimate peripheral participation Cambridge University Press. Cambridge UK.

Lave, J. and Wenger, E., 1999. Legitimate peripheral participation. Learners, learning and assessment, London: The Open University.pp. 83-89.

Macluskey, M. and Durham, J., 2009. Oral surgery undergraduate teaching and experience in the United Kingdom: a national survey. European Journal of Dental Education, 13(1), pp.52-57.

Stelzle, F., Farhoumand, D., Neukam, F.W. and Nkenke, E., 2011. Implementation and validation of an extraction course using mannequin models for undergraduate dental students. Acta Odontologica Scandinavica, 69(2), pp.80-87.

Wilson N. 20110ral surgery services and training. British Dental Journal. 210(3):pp.95-95. 